\title{
SITUACIÓN DE LA RESISTENCIA ANTIFÚNGICA DE ESPECIES DEL GÉNERO Candida EN PERÚ
}

\author{
Susana Zurita Macalupú ${ }^{1, a}$
}

\begin{abstract}
RESUMEN
Actualmente asistimos a un cambio constante y continuo del panorama epidemiológico de la resistencia de levaduras a antifúngicos, relacionado con el tipo de huésped, la etiología de la enfermedad, el microorganismo involucrado y sus mecanismos de resistencia. Esta situación epidemiológica hace más complicado el manejo de los pacientes con infecciones del torrente sanguíneo por especies del género Candida. Observándose una alta mortalidad, incremento en el uso de antifúngicos, así como el desarrollo de métodos fiables para realizar estudios de sensibilidad. Por tanto, es importante la vigilancia local y regional para conocer el perfil de sensibilidad y la distribución de especies de Candida, con la finalidad de instaurar una terapia antifúngica adecuada.
\end{abstract}

Palabras clave: Candida albicans; Candidemia; Resistencia fúngica a drogas; Fluconazol; Voriconazol (fuente: DeCS BIREME)

\section{SITUATION OF ANTI-FUNGAL RESISTANCE OF SPECIES OF THE GENUS Candida IN PERU}

\begin{abstract}
We are currently witnessing a constant and ongoing change of the epidemiological pictures of yeast resistance to antifungal agents, related to the type of host, etiology of the disease, the microorganism involved, and its mechanisms of resistance. This epidemiological situation complicates management of patients with infections of the bloodstream due to species of the genus Candida, exhibiting a high mortality and an increase in the use of anti-fungal agents, as well as the need to develop reliable methods to conduct sensitivity studies. Therefore, local and regional monitoring is important in order to know the sensitivity profile and the distribution of Candida species so as to start an adequate anti-fungal therapy.
\end{abstract}

Keywords: Candida albicans; Candidemia; Drug resistance, fungal; Fluconazole; Voriconazole (source: MeSH NLM)

\section{INTRODUCCIÓN}

Las infecciones fúngicas han incrementado en frecuencia e importancia en las últimas décadas, acompañadas de una alta mortalidad, generalmente ocasionadas por infecciones del torrente sanguíneo; causadas por especies del género Candida, en un $20 \%$ a $50 \%$ de los casos, por infecciones del género Aspergillus en un $40 \%$ a $80 \%$ de los casos y por algunos hongos emergentes. La mortalidad por estas infecciones puede ir más allá de $90 \%{ }^{(1,2)}$.

Estas cifras están determinadas por diversos factores relacionados con el huésped, la etiología de la enfermedad, el microorganismo y la resistencia o sensibilidad del antifúngico. Las poblaciones susceptibles de infecciones fúngicas oportunistas se han ido incrementando, debido a los avances médicos (uso inmuno-supresores y trasplante de órganos) que han permitido mejorar el pronóstico de muchas enfermedades, y por la aparición del VIH/SIDA, constituyéndose en uno de los principales grupos de riesgo para adquirir una micosis.

En Perú, en un reciente estudio en pacientes con VIH/SIDA, se estimó un total de 581174 casos de enfermedades fúngicas, de los cuales 1557 casos correspondieron a candidemia y 1621 casos a aspergilosis invasiva. Sin embargo, estas cifras pueden estar subestimadas, debido a que las enfermedades fúngicas, no son de notificación obligatoria, además de la ausencia de un sistema de vigilancia epidemiológica ${ }^{(3)}$.

En general, podemos decir que la incidencia anual de las infecciones fúngicas, dependiendo del tipo de infección e investigación realizada ha aumentado de 3 a 20 veces a partir de la década del $70^{(4,5)}$.

Estas infecciones oportunistas son principalmente nosocomiales y las especies del género Candida representan más del $80 \%$ de todas las infecciones, siendo la candidemia

\footnotetext{
Laboratorio de Referencia Nacional de Micología, Instituto Nacional de Salud. Lima, Perú

a Médico especialista en Enfermedades Infecciosas y Tropicales

Recibido: 14/01/2018 Aprobado: 21/02/2018 En línea: 23/03/2018
} 
una importante causa de enfermedad del torrente sanguíneo, que causa del $10 \%$ al $15 \%$ de las sepsis nosocomiales y prolonga hasta tres semanas la estancia hospitalaria, duplicando el riesgo de muerte del enfermo ${ }^{(5)}$.

La epidemiología de la sepsis en los Estados Unidos (EUA) muestra que el número anual de casos de etiología fúngica, aumentó en 207 \% en el período de 1979 a 2000, siendo el género Candida el principal agente etiológico ${ }^{(6)}$, asimismo, del 2008 al 2009 se reportaron 1752 casos de candidemia y el $44,5 \%$ de ellos ocurrieron en las Unidades de Cuidados Intensivos (UCI).

En Brasil, en un estudio multicéntrico de vigilancia en 16 hospitales (2007-2010), se encontró candidemia en 2,49 casos de cada 1000 admisiones hospitalarias, con una mortalidad bruta del $72 \%$ (53\% para pacientes que no estaban en la $\mathrm{UCl}$ y $85 \%$ para pacientes que estaban en UCI) ${ }^{(7,8)}$

En Perú se han realizado pocos estudios sobre candidemia, uno realizado en un hospital nacional de alta complejidad de Lima (2004-2006), reveló que el género Candida se encontró en el $11,6 \%$ de las muestras de hemocultivo de pacientes de $\mathrm{UCl}{ }^{(9)}$. Otro realizado en nueve hospitales de Lima (2009-2011), reportó 153 especies del género Candida que causaron candidemia ${ }^{(10)}$ y uno realizado en tres hospitales de Lima y Callao (2013 al 2015) reportó 158 aislamientos y una incidencia de candidemia por centro entre 1 y 2,6 casos por 1000 ingresos ${ }^{(11)}$.

Entre los factores de riesgo que propician la candidemia se incluyen la terapia antibiótica de amplio espectro, la permanencia en la $\mathrm{UCl}$ por más de 72 horas, la terapia inmunosupresora, la nutrición parenteral y los procedimientos médicos invasivos múltiples ${ }^{(12)}$. Las condiciones asociadas con mayor frecuencia a candidemia, en tres hospitales de Lima y Callao fueron: la cirugía reciente $(38,9 \%)$, la ventilación mecánica $(38,2 \%)$ y la nutrición parenteral total $(36,3 \%)^{(11)}$

En tal contexto, para el control de estas infecciones fúngicas, se identifican como herramientas fundamentales, el obtener un diagnóstico precoz, caracterizar las especies del género Candida y detectar la resistencia fúngica a drogas.

\section{IMPORTANCIA DE LA CARACTERIZACIÓN DE ESPECIES DEL GÉNERO Candida Y SU RELACIÓN EPIDEMIOLÓGICA EN LA RESISTENCIA FÚNGICA A DROGAS}

Existen aproximadamente 163 especies pertenecientes al género Candida y 10 de ellas son responsables de la mayoría de las infecciones fúngicas, siendo Candida albicans la especie más importante. Son microrganismos comensales en el hombre y colonizan mucosas del tracto intestinal (50\% a $70 \%)$, boca (30\% a $50 \%$ ), vagina (5\% a $30 \%$ ) y piel (4\% a $7 \%$ ). Su presencia en las manos en personal médico es de $20 \%$ y hasta $80 \%$ en personal de enfermería ${ }^{(13)}$.

Se reporta baja sensibilidad ( $40 \%$ a $75 \%)^{(26)}$ hemocultivos en enfermos con candidemia y en la mayoría de los casos el diagnóstico es presuntivo, ello conduce a una hospitalización prolongada, alta tasa de mortalidad, de alrededor del $50 \%$, y en una carga financiera para los sistemas de salud. Actualmente hay avances en el diagnóstico laboratorial de candidemia mediante la detección de antígenos, y material genético, utilizando pruebas moleculares. Sin embargo, estas no están a disposición de la gran mayoría de hospitales y se mantiene aún como parámetros de diagnóstico presuntivo el grado de sospecha, la valoración de los factores de riesgo, síntomas y signos clínicos, así como la presencia de colonización por Candida en otros sitios ${ }^{(14)}$. Debido a esta dificultad en el diagnóstico, la incidencia real de candidemia es poco conocida y puede variar a nivel institucional y regional.

A esto se suma, el uso masivo de antifúngicos tanto en forma preventiva como terapéutica, lo que ha originado la aparición de resistencias secundarias y probablemente el desplazamiento de cepas sensibles por otras más resistentes.

Asimismo, encontramos un cambio del patrón etiológico de la candidemia, a pesar de continuar presentándose la Candida albicans como la especie más frecuente, su prevalencia ha disminuido drásticamente, lo que da lugar a un aumento de las Candida no albicans. Estas especies no albicans generan dificultades terapéuticas, emergiendo con ellas nuevos patrones de sensibilidad a los antifúngicos.

Con la introducción del fluconazol, en los años ochenta, las candidemias por Candida albicans y Candida tropicalis disminuyeron. Sin embargo, las causadas por Candida krusei y principalmente por Candida glabrata experimentaron un aumento significativo, debido a que una de ellas posee resistencia intrínseca a fluconazol (Candida krusei) y la otra elevada resistencia a este fármaco (Candida glabrata) ${ }^{(13)}$.

En el estudio de vigilancia de resistencia ARTEMIS (19972005), que incluyó aislados provenientes de la región del Pacífico/Asia, Latinoamérica, Europa, Oriente Medio/África y Norteamérica, por un periodo de 8,5 años, se observó el incremento de resistencia al fluconazol: Candida albicans (0,8 \% a 1,5\%); Candida tropicalis (3 \% a 6,6 \%); Candida parapsilosis (2,0 \% a 4,2 \%); Candida Iusitaniae (1,6 \% a $6,6 \%$ ) y Candida kefyr (0 \% a $5,7 \%)^{(14)}$. La resistencia informada al fluconazol en especies de Candida albicans es cercana al $3 \%$, y los reportes acerca esta resistencia, tienen variaciones regionales y locales muy notorias ${ }^{(16)}$. 
En Brasil, los estudios epidemiológicos sobre candidemia, mostraron que las especies de Candida no albicans representan el 65,7 \% (Candida parapsilosis 24,1\%, Candida tropicalis $15,3 \%$ y Candida glabrata 10,2 \%) y para Candida albicans 34,3\%. La resistencia a fluconazol se limitó a Candida krusei y Candida glabrata. Todas fueron susceptibles a anidulafungina y anfotericina B (17). En el resto de América Latina las especies de Candida que producen candidemia se distribuyen de la siguiente manera: Candida albicans $50 \%$, Candida tropicalis $20 \%$ y Candida parapsilosis $16 \%{ }^{(18,19)}$.

En el Perú, son pocos los estudios realizados, un reporte de un hospital nacional (2004-2006), señaló como especies causantes de candidemia a Candida albicans $39 \%$ y Candida no albicans $61 \%$ (Candida tropicalis $26 \%$; Candida parapsilosis $10 \%$; Candida guillemondi $10 \%$ y Candida glabrata $4 \%$, entre otras) ${ }^{(9)}$. Otro estudio realizado en nueve hospitales nacionales (2009-2011) reveló el predominio de las especies no albicans 60,1\% (Candida parapsilosis $28 \%$; Candida tropicalis $24 \%$; Candida glabrata $5 \%$; Candida guillermondii $2 \%$; Candida krusei $0,7 \%$ y Candida famata $0,7 \%$ ) y Candida albicans $39,9 \%$. Entre las especies no Albicans; $98 \%$ fueron susceptibles a anfotericina B, 98,7 \% a fluconazol, $98 \%$ a voriconazol y $97,4 \%$ anidulafungina ${ }^{(10)}$. En el período 2012 al 2013, el Laboratorio Referencia Nacional de Micología del Instituto Nacional de Salud (INS) de Perú, con la participación de siete hospitales nacionales, reportó que las especies más frecuentes fueron Candida albicans $66,6 \%$; Candida tropicales $29,6 \%$ y Candida parapsilosis $11,1 \%$. Entre las especies poco frecuentes se encontraron a Candida lusitania 7,4 \%; Candida krusei 7,4 \%; Candida zeylanoides $3,7 \%$ y Candida guillermondii 3,7 \%. La concentración mínima inhibitoria $50 \%$ y $90 \%$ en $\mathrm{mg} / \mathrm{L}$ fue de 0,25 y 0,5 para la anfotericina B; 1 y 4 para fluconazol; 0,03 y 0,25 para el Itraconazol; 0,03 y 0,12 para voriconazol y 0,25 y 2 por caspofungina. La especie menos susceptible fue Candida guillermondii. (20).

Un estudio realizado en tres hospitales de Lima y Callao (2013-2015), informó que las especies Candida no albicans representaron el 71,9\% (Candida parapsilosis 25,3\%; Candida tropicalis 24,7\%; Candida glabrata 9,5\%; Candida guillermondii 7,0 \%; Candida krusei 0,6\% y otras 5,1\%) y Candida albicans $27,8 \%$. Se encontró cuatro aislamientos resistentes a fluconazol $(2,6 \%)$ y 17 fueron sensibles dosis dependiente a fluconazol (10,7 \%). También se observó disminución en la susceptibilidad en tres aislados al posaconazol y uno a voriconazol. Todos los aislados fueron

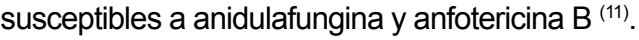

Los estudios de candidemia realizados en América Latina y Perú indican resultados similares, respecto a que la Candida krusei no es frecuentemente aislada y que la resistencia a fluconazol es baja y varía según la especie de Candida (Tabla 1)

Tabla 1. Resistencia a fluconazol / voriconazol según especies de Candida en diversos estudios

\begin{tabular}{|c|c|c|c|c|c|c|c|c|c|}
\hline \multirow{2}{*}{ País/Región } & \multirow{2}{*}{$\mathbf{n}$} & \multicolumn{7}{|c|}{ Especies de Candida n (\%) } & \multirow{2}{*}{$\begin{array}{l}\text { *Resistencia fluconazol, } \\
\text { voriconazol n (\%) }\end{array}$} \\
\hline & & C. albicans & C.parapsilosis & C. tropicalis & C. guillermondii & C.glabrata & C. krusei & otras & \\
\hline $\begin{array}{l}\text { Asia/Pacifico, } \\
\text { Europa, África, } \\
\text { América } \\
\text { Latina, Norte } \\
\text { América }^{(15)}\end{array}$ & 10337 & $6443(62,0)$ & NR & $1816(18,0)$ & NR & $2078(20,0)$ & NR & NR & $\begin{array}{l}\text { Fluconazol: C. albicans } 90(1,4) \text {, } \\
\text { C. glabrata } 347(16,7) \text {, C. tropicalis } \\
73(4,0) \text {. } \\
\text { Voriconazol: C. albicans } 77(1,2), \text { C. } \\
\text { glabrata } 245(11,8), \\
\text { C. tropicalis } 99(5,5) .\end{array}$ \\
\hline $\begin{array}{l}\text { América } \\
\text { Latina }^{(18)}\end{array}$ & 672 & $253(37,6)$ & $178(26,5)$ & $118(17,6)$ & $44(6,5)$ & $42(6,3)$ & $18(2,7)$ & $19(2,8)$ & $\begin{array}{l}\text { Fluconazol:C. glabrata } 3(7,1) \text {, } \\
\text { C.krusei } 18(100,0) \\
\text { Voriconazol: NR }\end{array}$ \\
\hline $\begin{array}{l}\text { América } \\
\text { Latina }^{(20)}\end{array}$ & 521 & $142(27,2)$ & $208(39,9)$ & $95(18,2)$ & $28(5,3)$ & NR & NR & $48(9,0)$ & $\begin{array}{l}\text { Fluconazol: C. albicans } 1(0,7) \text {, } \\
\text { C. parapsilosis } 6(2,9) \text {. } \\
\text { Voriconazol: C. parapsilosis } \\
1(0,5), \text { C. tropicalis } 5(5,2)\end{array}$ \\
\hline Perú (9) & 51 & $20(39)$ & $5(10,0)$ & $13(26,0)$ & $5(10,0)$ & $2(4,0)$ & 0 & $6(11,0)$ & NR \\
\hline Perú ${ }^{(10)}$ & 153 & $61(39,9)$ & $43(28,1)$ & $36(23,5)$ & $3(2,0)$ & $8(5,2)$ & $1(0,7)$ & $1(0,7)$ & $\begin{array}{l}\text { Fluconazol: C. parapsilosis } 1(2,3) \text {. } \\
\text { Voriconazol: C. albicans } 3(5,0)\end{array}$ \\
\hline Perú ${ }^{(11)}$ & 158 & $44(27,8)$ & $40(25,3)$ & $39(24,7)$ & $11(7,0)$ & $15(9,5)$ & $1(0,6)$ & $8(5,1)$ & $\begin{array}{l}\text { Fluconazol: C. albicans } 1(2,2) \text {, } \\
\text { C. parapsilosis } 2(5,0), \text { C. krusei } 1 \\
(100,0) \text {. } \\
\text { Voriconazol: NR }\end{array}$ \\
\hline
\end{tabular}


También debe tenerse en cuenta la aparición de Candida auris, especie emergente a nivel global, multiresistente, causante de infecciones fúngicas invasoras asociadas a altas tasas de mortalidad ${ }^{(27)}$ y que aún no ha sido reportado en el Perú.

Los pocos estudios realizados en Perú mencionan que la candidemia se produce por especies sensibles in vitro al fluconazol, que es el antifúngico más utilizado, por lo que es correcto utilizar fluconazol como tratamiento inicial en candidemia. Sin embargo, si se identifica la cepa causante como Candida krusei, una especie resistente a este antifúngico debe recomendarse un cambio de tratamiento. Si la cepa causante es Candida albicans y al realizar un estudio de sensibilidad la concentración mínima inhibitoria CMI, indica resistente de fluconazol, se aconseja un cambio de terapia. Asimismo, tener en cuenta la aparición persistente de especies de Candida no albicans como Candida parapsilosis, Candida glabrata y Candida guillermondii que resultaron resistentes a fluconazol. Otro punto importante es la necesidad de correlacionar los resultados de sensibilidad con las respuestas clínicas al tratamiento.

En todas las situaciones mencionadas, se requiere de la caracterización de especies del género Candida y conocer el perfil de susceptibilidad para instaurar el tratamiento adecuado.

Tambien es importante señalar, la opinión de la mayoría de expertos que indican, que las pruebas de sensibilidad antifúngica en la detección de resistencia adquirida, juega un papel primordial y tiene utilidad práctica, sin embargo, otros opinan que no ayuda en el manejo de pacientes con micosis invasora, sea por problemas de estandarización de los métodos y porque las alternativas terapéuticas son escasas para combatir la micosis invasora y por tal razón, las pruebas de sensibilidad no tendrían mucho sentido en la práctica clínica.

Sin embargo, en la actualidad contamos con dos estándares para la determinación de resistencia antifúngica; el Clinical and Laboratory Standards Institute (CLSI) en EUA y el European Committee on Antimicrobial Susceptibility Testing (EUCAST) en Europa, que permite la detección de resistencia. El impacto de ambas es haber logrado la estandarización del método de susceptibilidad in vitro para levaduras, al aumentar la concordancia entre laboratorios (es decir, son reproducibles y fiables), además de estandarizarlas como técnicas de referencia. Ambos métodos CLSI y EUCAST utilizan la microdilución en caldo, pero difieren en el tamaño del inóculo y en los puntos de corte para interpretar la susceptibilidad. Ambos son concordantes en la medición de la CMI de azoles y equinocandinas contra Candida y discriminan cepas susceptibles silvestres (que no han adquirido resistencia) y cepas resistentes (que exhiben mecanismos de resistencia intrínseca o adquirida) ${ }^{(21-24)}$.

El CLSI también ha estandarizado la medición de susceptibilidad mediante difusión en disco, para fluconazol y voriconazol contra Candida ${ }^{(23)}$. Actualmente los métodos comerciales aprobados por la FDA y validados con el método CLSI son Sensititre YesOne colorimetric plate ${ }$, Vitek 2 yeast susceptibility test $\circledast$ y Etest $\circledast$.

En el Perú el INS ha estandarizado el método de referencia del CLSI utilizando la microdilución en caldo y ha transferido a la red nacional de laboratorios de micología, el método de difusión en disco del CLSI para fluconazol y voriconazol. La concordancia entre estos laboratorios es evaluada, mediante el programa de evaluación de desempeño de la calidad. Respecto a los métodos comerciales mencionados, estos son utilizados en algunos hospitales nacionales de alta complejidad, ubicados en Lima.

Tanto el CLSI como el EUCAST han establecido puntos de corte clínicos (en base a distribuciones de la CMI, parámetros farmacocinéticos y farmacodinámicos, mecanismos de resistencia y respuesta clínica) y epidemiológicos (epidemiologic cutoff values o ECVs). Estos puntos de corte son revisados continuamente en relación con la aparición de nuevos fármacos y de nuevos mecanismos de resistencia antifúngica, que pueden ser primarios o secundarios y que dependen de las características intrínsecas o adquiridas de los patógenos fúngicos.

Los mecanismos de resistencia antifúngica continúan evolucionando y aumentando, los más conocidos y estudiados indican una resistencia adquirida a azoles, inducidas por bombas de eflujo codificadas por los genes $M D R$ o $C D R$, y la adquisición de mutaciones puntuales en el gen que codifica para la enzima blanco de estos fármacos (gen ERG11). Si hay sobreexpresión de bombas de eflujo y mutaciones de ERG11, el nivel de resistencia a voriconazol y fluconazol es mucho más alto (efecto aditivo). Respecto a la resistencia adquirida de especies de Candida a equinocandinas, se ha identificado mutaciones en los genes $F K S$ que codifican la subunidad mayor de la enzima blanco de estos antifúngicos (1,3- $\beta$-D glucan sintetasa) ${ }^{(24,25)}$. Al respecto cepas peruanas que intervinieron en un estudio multilaboratorio reveló que las mutaciones en el gen ERG11 y la sobrexpresión de bombas de eflujo codificada por los genes CDR puede explicar el mecanismo de resistencia al fluconazol (28).

En general, podemos mencionar que la detección de resistencia a los antifúngicos y la investigación de nuevos mecanismos de resistencia nos permite cambiar o ajustar las terapias de acuerdo con la sensibilidad, desarrollar nuevos fármacos y modificar los ya existentes. 


\section{CONCLUSIÓN}

Actualmente estamos frente a un cambio constante y continuo del panorama epidemiológico de la resistencia de levaduras a antifúngicos, relacionado con el tipo de huésped, la etiología de la enfermedad, el microorganismo involucrado y sus mecanismos de resistencia, haciendo más complicado el manejo de los pacientes con candidemia. Para tal situación, el obtener un diagnóstico precoz, caracterizar las especies de Candiday detectar la resistencia fúngica a drogas, se constituyen en herramientas básicas, para el control de esta micosis invasora. Actualmente contamos con métodos fiables para realizar estudios de sensibilidad antifúngica, hecho importante en la vigilancia epidemiológica local y regional, pues permiten conocer el perfil de sensibilidad y la distribución de especies, para instaurar el tratamiento adecuado.

Agradecimientos: A la bióloga Flor Urcia y a la técnica Alida Navarro por la recolección de datos.

Fuente de Financiamiento: Autofinanciado.

Conflictos de interés: La autora declara no tener conflicto de interés.

\section{REFERENCIAS BIBLIOGRÁFICAS}

1. Lortholary O, Renaudat C, Sitbon K, Madec Y, Denoeud-Ndam L, Wolff M, et al. Worrisome trends in incidence and mortality of candidemia in intensive care units (Paris area, 2002-2010). Intensive Care Med. 2014;40(9):1303-12. doi: 10.1007/s00134-014-3408-3.

2. Bassetti M, Righi E, Ansaldi F, Merelli M, Trucchi C,DePascaleG, et al. A multicenter study of septic shock due to candidemia: outcomes and predictors of mortality. Intensive Care Med. 2014;40(6):839-45. doi: 10.1007/s00134-014-3310-z.

3. Bustamante B, Denning D, Campos P. Serious fungal infections in Peru. Eur J Clin Microbiol Infect Dis. 2017;36(6):943-948. doi: 10.1007/s10096-017-2924-9.

4. Guarro J, Kantarcioglu A, Horré R, Rodriguez-Tudela J, Cuenca-Estrella $\mathrm{M}$, Berenguer J, et al. Scedosporium apiospermum: changing clinical spectrum of a therapy-refractory opportunist. Med Mycol. 2006;44(4):295-327. doi: $10.1080 / 13693780600752507$.

5. Cuenca-Estrella M, Rodríguez-Tudela JL, Córdoba S, Melhem MC, Szeszs MW, Castañeda E, et al. Red Regional de Laboratorios para la Vigilancia de las Infecciones Fúngicas Invasoras $\mathrm{y}$ Susceptibilidad a los Antifúngicos. Rev Panam Salud Publica. 2008;23(2):12934.

6. Pfaller MA, Diekema DJ. Epidemiology of Invasive Candidiasis: a Persistent Public Health Problem. Clin Microbiol Rev. 2007;20(1):133-63. doi: 10.1128/ CMR.00029-06.

7. Colombo A, Nucci M, Park B, Nouér $S$, Arthington-Skaggs B, da Matta D, et al. Epidemiology of Candidemia in Brazil: a Nationwide Sentinel Surveillance of Candidemia in Eleven Medical Centers. J
Clin Microbiol. 2006; 44(8):2816-2823. doi: 10.1128/JCM.00773-06.

8. Doi A, Campos Pignatari A, Edmond M, Rodrigues Marra A, Aranha Camargo L, Siqueira R, et al. Epidemiology and Microbiologic Characterization of Nosocomial Candidemia from a Brazilian National Surveillance Program. PLoS One. 2016;11(1):e0146909. doi: 10.1371/journal.pone.0146909.

9. Paz Rojas EL, de León Pandolfi DP, Ramírez Ponce R. Resistencia bacteriana en cuidados intensivos y tendencia actual: Departamento de Cuidados Críticos, Servicio de Cuidados Intensivos del Hospital Nacional Guillermo Almenara Irigoyen, Essalud, Lima, Perú, 20042006. Acta Med Per. 2008; 25(3):140147.

10. Bustamante B, Martins M, Bonfietti L, Szeszs M, Jacobs J, Garcia C, et al. Species distribution and antifungal susceptibility profile of Candida isolates from bloodstream infections in Lima, Perú. J Med Microbiol. 2014; 63, 855-860. doi: 10.1099/jmm.0.071167-0.

11. Rodriguez L, Bustamante B, Huaroto L, Agurto C, Illescas R, Ramirez R, et al. A multi-centric Study of Candida bloodstream infection in Lima-Callao, Peru: Species distribution, antifungal resistance and clinical outcomes. PLoS One. 2017;12(4):e0175172. doi: 10.1371/journal.pone.0175172.

12. Serrano R, Gimeno A, Plumed L, Pemán J, Álvarez B, Plazas J, et al. Perfil epidemiológico y patrón de sensibilidad de aislamientos causantes de infección fúngica invasora frente a aislamientos fúngicos de colonización en pacientes críticos no neutropénicos. Rev Iberoam Micol. 2013;30(1):14-20. doi: 10.1016/j. riam.2012.06.003.
13. Silva V, Díaz MC, Febré N. Vigilancia de la resistencia de levaduras a antifúngicos. Rev Chil Infect. 2002;19(S2), 149-156. doi: 10.4067/S0716-10182002019200016.

14. Tobar E, Silva F, Olivares R, Gaete P, Luppi M. Candidiasis invasoras en el paciente crítico adulto. Rev Chil Infect. 2011; 28(1): 41-49. doi: 10.4067/ S0716-10182011000100008.

15. Pfaller MA, Diekema DJ, Gibbs DL, Newell VA, Meis JF, Gould IM, et al. Results from the ARTEMIS DISK Global Antifungal Surveillance Study, 1997 to 2005: an 8.5-Year Analysis of Susceptibilities of Candida Species and Other Yeast Species to Fluconazole and Voriconazole Determined by CLSI Standardized Disk Diffusion Testing. J Clin Microbiol. 2007; 45(6): 1735-1745. doi: 10.1128/JCM.00409-07.

16. Gómez Quintero $\mathrm{CH}$. Resistencia de levaduras del genero Candida al fluconazol. Infectio. 2010;14(S2): S172-S180.

17. Nucci M, Queiroz-Telles F, Tobón A, Restrepo A, Colombo A. Epidemiology of Opportunistic Fungal Infections in Latin America. Clin Infect Dis. 2010;51(5):56170. doi: $10.1086 / 655683$.

18. Nucci M, Queiroz-Telles F, AlvaradoMatute T, Tiraboschi IN, Cortes J, Zurita $\mathrm{J}$, et al. Epidemiology of Candidemia in Latin America: A Laboratory-Based Survey. PLoS ONE. 2013; 8(3): e59373.

19. del Palacio A, Villar J, Alhambra A Epidemiología de las candidiasis invasoras en población pediátrica y adulta. Rev Iberoam Micol. 2009 Mar 31;26(1):2-7. doi: 10.1016/S1130-1406(09)70002-6.

20. Córdoba S, Melhem M, Dolande M, Puimé A, Martínez G, Zurita S, et al. Species Distribution and Susceptibility Profile of Yeasts Isolated from Blood Cultures: Pre- 
liminary Results of a Multicenter Study for Latin America. En: 52nd Annual ICAAC. San Francisco, CA; 2012.

21. Cuenca-Estrella M, Rodriguez-Tudela J. The current role of the reference procedures by CLSI and EUCAST in the detection of resistance to antifungal agents in vitro. Expert Rev Anti Infect Ther. 2010; 8(3):267-276. doi: 10.1586/eri.10.2.

22. Rex JH, Alexander BD, Andes D, Arthington - Skaags B, Brown SD, Chaturvedi V, et al. Reference Method for Broth Dilution Antifungal Susceptibility Testing of Yeasts: Approved Standard. (CLSI Document M27-A3). 3rd ed. Wayne, PA: Clinical and Laboratory Standards Institute; 2008.

23. Clinical and Laboratory Standards Institute. Zone diameter interpretative standards, corresponding minimal inhibitory concentration (MIC) interpretative breakpoints, and quality control limits for antifungal disk diffusion susceptibility testing of yeasts; informational supplement. (CLSI document M44-S3). 3rd ed. Villanova, PA: Clinical and Laboratory Standards Institute; 2009.

24. Pfaller M. Antifungal drug resistance: Mechanisms, Epidemiology, and consequences for treatment. Am J Med. 2012;125(1 Suppl):S3-13. doi: 10.1016/j.amjmed.2011.11.001.

25. López-Ávila K, Dzul-Rosado K, LugoCaballero C, Arias-León J, Zavala-Castro J. Mecanismos de resistencia antifúngica de los azoles en Candida albicans. Una revisión. Rev Biomed 2016; 27:127-136.

26. Figueras C, Díaz de Heredia C, García JJ, Navarro M, Rossich R, Rumbao $\mathrm{J}$, et al. Recomendaciones de la Sociedad Española de Infectología Pediátrica sobre diagnóstico y tratamiento de la candidiasis invasiva. An Pediatr (Barc). 2011;74(5):337.e1-337.e17. doi: 10.1016/j.anpedi.2010.12.012.
27. Larkin E, Hager C, Chandra J, Mukherjee P, Retuerto M, Salem I, et al. The Emerging Pathogen Candida auris: Growth Phenotype, Virulence Factors, Activity of Antifungals, and Effect of SCY-078, a Novel Glucan Synthesis Inhibitor, on Growth Morphology and Biofilm Formation. Antimicrob Agents Chemother. 2017;61(5). pii: e02396-16. doi: 10.1128/AAC.02396-16.

28. Espinel-Ingroff A, Pfaller MA, Bustamante B, Canton E, Fothergill A, Fuller J, et al. Multilaboratory Study of Epidemiological Cutoff Values for Detection of Resistance in Eight Candida Species to Fluconazole, Posaconazole, and Voriconazole. Antimicrob Agents Chemother.2014;58(4):200612. doi: 10.1128/AAC.02615-13.

Correspondencia: Susana Zurita Macalupú Dirección: Calle Cápac Yupanqui 1400. Lima 11, Perú

Teléfono: (511) 748-1111 anexo 2140

Correo electrónico:szurita@ins.gob.pe

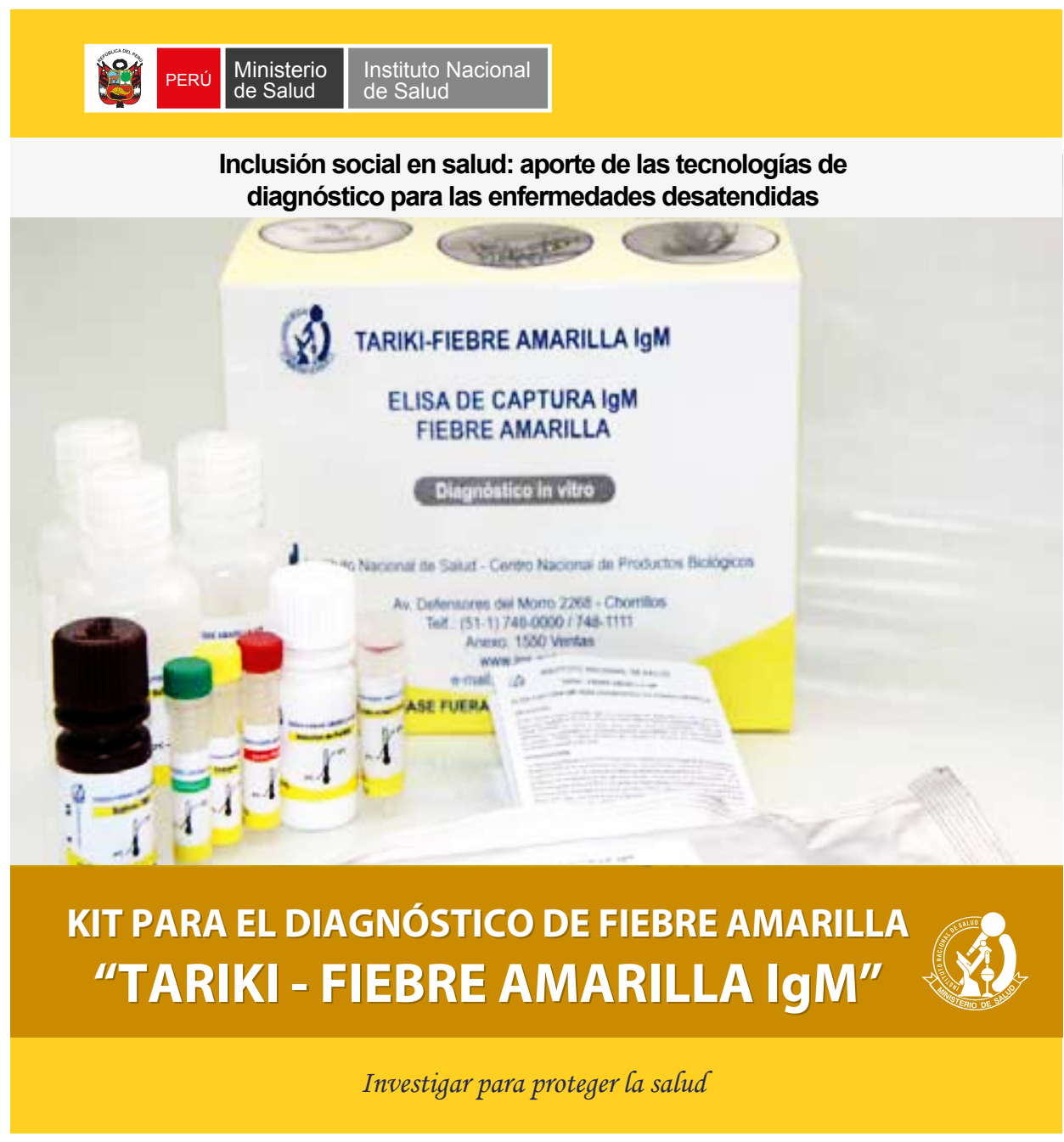

